PROCEEDINGS OF THE

AMERICAN MATHEMATICAL SOCIETY

Volume 139, Number 4, April 2011, Pages 1495-1510

S 0002-9939(2010)10830-4

Article electronically published on December 8, 2010

\title{
CHARACTERIZING ISOTOPIC CONTINUA IN THE SPHERE
}

\author{
LEX G. OVERSTEEGEN AND KIRSTEN I. S. VALKENBURG
}

(Communicated by Alexander N. Dranishnikov)

\begin{abstract}
In this paper we will generalize the following well-known result. Suppose that $I$ is an arc in the complex sphere $\mathbb{C}^{*}$ and $h: I \rightarrow \mathbb{C}^{*}$ is an embedding. Then there exists an orientation-preserving homeomorphism $H$ : $\mathbb{C}^{*} \rightarrow \mathbb{C}^{*}$ such that $H\lceil I=h$. It follows that $h$ is isotopic to the identity.

Suppose $X \subset \mathbb{C}^{*}$ is an arbitrary, in particular not necessarily locally connected, continuum. In this paper we give necessary and sufficient conditions on an embedding $h: X \rightarrow \mathbb{C}^{*}$ to be extendable to an orientation-preserving homeomorphism of the entire sphere. It follows that in this case $h$ is isotopic to the identity. The proof will make use of partitions of complementary domains $U$ of $X$, into hyperbolically convex subsets, which have limited distortion under the conformal map $\varphi_{U}: \mathbb{D} \rightarrow U$ on the unit disk.
\end{abstract}

\section{INTRODUCTION}

The question which embeddings of subcontinua of manifolds are isotopic to the identity has been extensively studied. Starting with the Schoenflies Theorem that every two simple closed curves in the plane are equivalently embedded, many papers have been devoted to a proof of this theorem and the similar result that any homeomorphism between two simple closed curves in the plane can be extended to a homeomorphism of the entire plane. See 18 for a recent proof and some history on this problem. In general strong conditions are necessary (see, for example, 8] for sufficient conditions on the existence of isotopies in higher dimensions). Bing and Starbird [3, Theorem 7.3] showed when two linear embeddings of a triangulated complex into the plane are isotopic. It was shown in [15] that an isotopy of a plane continuum $h: X \times[0,1] \rightarrow \mathbb{C}$, which starts at the identity, can be extended to an isotopy of the entire plane. In this paper we will give sufficient conditions for the existence of an extension of an embedding of an arbitrary continuum $X$ in the sphere to a homeomorphism of the entire sphere. From this we will be able to conclude when such an embedding is isotopic to the identity.

Suppose $X$ is a continuum in the sphere $\mathbb{C}^{*}, h: X \rightarrow Y \subset \mathbb{C}^{*}$ is a homeomorphism and $U$ is a complementary domain of $X$. If we want to extend $h$ homeomorphically over $U$ it is clearly necessary that, given a point $z \in X$ which

Received by the editors November 2, 2009 and, in revised form, April 8, 2010.

2010 Mathematics Subject Classification. Primary 54C20, 57N37; Secondary 57N05.

Key words and phrases. Isotopy, homeomorphic extension, isotopic continua.

The first author was supported in part by NSF-DMS-0906316.

The second author was supported by the Netherlands Organisation for Scientific Research (NWO), under grant 613.000.551, and thanks the Department of Mathematics at UAB for its hospitality.

(C) 2010 American Mathematical Society
Reverts to public domain 28 years from publication 1495 
is accessible from a complementary domain $U$ of $X, h(z)=w$ is accessible from a complementary domain $V$ of $Y$. In other words, the homeomorphism $h: X \rightarrow Y$ must induce homeomorphisms $\hat{h}_{U}: S_{U}^{1} \rightarrow S_{V}^{1}$, where $U$ and $V$ are corresponding complementary domains of $X$ and $Y$ and $S_{U}^{1}$ and $S_{V}^{1}$ are the circles of prime ends of $U$ and $V$, respectively. However, as we will show, this by itself is not sufficient. (It will follow as a corollary to our main result that this condition is sufficient if $X$ is a locally connected continuum.)

Early results relied on the conformal theory and are especially well-suited to address the case when $X$ is locally connected. Given a simply connected domain $U$ in the sphere such that $\partial U$ is a nondegenerate continuum, let $\varphi_{U}: \mathbb{D} \rightarrow U$ be a conformal map. In this case we can identify the unit circle $S_{U}^{1}=\partial \mathbb{D}$ with the circle of prime ends of $U$ (see [14]). We will identify points $\theta \in S_{U}^{1}$ by their arguments $\theta \in[0,2 \pi)$ and call them angles. If $\partial U$ is locally connected, then $\varphi_{U}$ extends continuously to a map $\overline{\varphi_{U}}: \overline{\mathbb{D}} \rightarrow \bar{U}$ providing a way to extend the map $h$ over $U$. However, if $\partial U$ is not locally connected, the map $\varphi_{U}$ is not uniformly continuous and this method is less promising. We will solve this problem by partitioning the disk into hyperbolically convex subsets $G_{\alpha}$ such that the family of maps $\left\{\varphi_{U}\left\lceil G_{\alpha}\right\}\right.$ is uniformly equicontinuous.

We denote by $\mathcal{A}_{U} \subset S_{U}^{1}$ the set of accessible angles: the set of angles such that the corresponding external ray $R_{\theta}=\varphi_{U}\left(\left\{r e^{\theta i} \mid 0 \leq r<1\right\}\right)$ lands on a point $x_{\theta} \in \partial U$ (i.e., $\overline{R_{\theta}} \backslash R_{\theta}=\left\{x_{\theta}\right\}$ ). We will consider the Mazurkiewicz metric $\rho_{U}$ on the set $\mathcal{A}_{U}$ which is defined as follows: for $\theta, \gamma \in \mathcal{A}_{U}, \rho_{U}(\theta, \gamma)$ is the infimum of the diameter of $\operatorname{arcs} \varphi_{U}(A)$, where $A \subset \mathbb{D}$ is an open $\operatorname{arc}$ from $\theta$ to $\gamma$. This notion plays an important role in our main result. As it is of independent interest as well, we will end this article by showing that $\mathcal{A}_{U}$, with the Mazurkiewicz metric, is always a separable, complete metric space. However, it can be topologically very different from a subset of the circle with the standard topology.

Throughout this paper we will assume that we are given a homeomorphism $h: X \rightarrow Y$ between two continua in the sphere. Let $U$ and $V$ be complementary domains of $X$ and $Y$, respectively. To avoid confusion, the conformal maps to $U$ and $V$, respectively, are denoted by $\varphi_{U}: \mathbb{D}_{U} \rightarrow U$ and $\varphi_{V}: \mathbb{D}_{V} \rightarrow V$. Similarly, we denote the respective sets of accessible angles by $\mathcal{A}_{U} \subset S_{U}^{1}=\partial \mathbb{D}_{U}$ and $\mathcal{A}_{V} \subset$ $S_{V}^{1}=\partial \mathbb{D}_{V}$. We can extend both conformal maps over the set of accessible angles $\mathcal{A}_{U}$ and $\mathcal{A}_{V}$, respectively, by defining for $\theta \in \mathcal{A}_{U}, \varphi_{U}(\theta)=x_{\theta}$ (for $\theta \in \mathcal{A}_{V}$, $\left.\varphi_{V}(\theta)=x_{\theta}\right)$ and we denote these extensions also by $\varphi_{U}: \mathbb{D}_{U} \cup \mathcal{A}_{U} \rightarrow U \cup \varphi_{U}\left(\mathcal{A}_{U}\right)$ and $\varphi_{V}: \mathbb{D}_{V} \cup \mathcal{A}_{V} \rightarrow V \cup \varphi_{V}\left(\mathcal{A}_{V}\right)$. It is well-known 14 that $\mathcal{A}_{U}$ and $\mathcal{A}_{V}$ are full measure subsets of $S^{1}$ and that the extended maps $\varphi_{U}$ and $\varphi_{V}$ are not necessarily continuous.

Definition 1.1. Given a homeomorphism $h: \partial U \rightarrow \partial V$, we say that a homeomorphism $\hat{h}: S_{U}^{1} \rightarrow S_{V}^{1}$ from the prime end circle of $U$ to the prime end circle of $V$ is the homeomorphism induced by $h$ or the induced homeomorphism provided that $\hat{h}\left\lceil\mathcal{A}_{U}: \mathcal{A}_{U} \rightarrow \mathcal{A}_{V}\right.$ is a bijection such that the following diagram commutes:

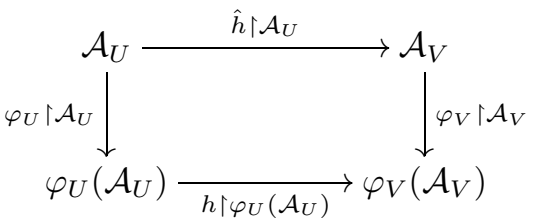


In this case we say that the domains $U$ and $V$ are corresponding complementary domains.

The following theorems are our main results.

Theorem 1.2. Suppose that $h: X \rightarrow Y$ is a homeomorphism between continua $X$ and $Y$ in the sphere $\mathbb{C}^{*}$ such that:

(1) there exists a bijection $\psi$ between the complementary components of $X$ and $Y$ such that for each component $U$ of $\mathbb{C}^{*} \backslash X, h\lceil\partial U$ induces a homeomorphism $\hat{h}_{U}: S_{U}^{1} \rightarrow S_{V}^{1}$ from the prime end circle $S_{U}^{1}$ of $U$ to the prime end circle $S_{V}^{1}$ of the complementary domain $V=\psi(U)$ of $Y$;

(2) the family $\left\{\hat{h}_{U}\left\lceil\mathcal{A}_{U}: \mathcal{A}_{U} \rightarrow \mathcal{A}_{V}\right\}\right.$, over all complementary domains $U$ of $X$, is uniformly equicontinuous in the Mazurkiewicz metric, where $V=$ $\psi(U)$.

Then $h$ extends to a homeomorphism $H: \mathbb{C}^{*} \rightarrow \mathbb{C}^{*}$.

Combining the above with known results, we get the following:

Theorem 1.3 (Main Theorem). Suppose that $h: X \rightarrow Y$ is a homeomorphism between two continua $X$ and $Y$ in the sphere. Then the following are equivalent:

(1) $h$ is isotopic to the identity on $X$;

(2) there exists an isotopy $F: \mathbb{C}^{*} \times[0,1] \rightarrow \mathbb{C}^{*}$ such that $F^{0}=\operatorname{id}_{\mathbb{C}^{*}}$ and $F^{1} \uparrow X=h$;

(3) $h$ extends to an orientation-preserving homeomorphism $H: \mathbb{C}^{*} \rightarrow \mathbb{C}^{*}$;

(4) (a) there exists a bijection $\psi$ between the complementary components of $X$ and $Y$ such that for each component $U$ of $\mathbb{C}^{*} \backslash X, h \uparrow \partial U$ induces a homeomorphism $\hat{h}_{U}: S_{U}^{1} \rightarrow S_{V}^{1}$ from the prime end circle $S_{U}^{1}$ of $U$ to the prime end circle of the complementary domain $\psi(U)=V$ of $Y$;

(b) there exists a complementary domain $U$ of $X$ such that the induced homeomorphism $\hat{h}_{U}: S_{U}^{1} \rightarrow S_{V}^{1}$ preserves the circular order;

(c) the family $\left\{\hat{h}_{U} \uparrow \mathcal{A}_{U}: \mathcal{A}_{U} \rightarrow \mathcal{A}_{V}\right\}$, over all complementary domains $U$ of $X$, is uniformly equicontinuous in the Mazurkiewicz metric.

Given a sequence of subsets $A_{n}$ of the sphere, we say that $\lim A_{n}=A_{\infty}$ provided $\lim d_{H}\left(\bar{A}_{n}, \bar{A}_{\infty}\right)=0$, where $d_{H}$ denotes the Hausdorff metric on the space of closed subsets of the sphere with the spherical metric.

\section{Preliminaries}

Denote the complex plane by $\mathbb{C}$, the origin by $O$, the open unit disk by $\mathbb{D}$, its boundary $\partial \mathbb{D}$ by $S^{1}$ and the complex sphere by $\mathbb{C}^{*}=\mathbb{C} \cup\{\infty\}$. Let $X$ be a proper subcontinuum in the sphere and $U$ a component of $\mathbb{C}^{*} \backslash X$. We may assume without loss of generality that $U$ contains the point $\infty$ at infinity. Let $\varphi_{U}: \mathbb{D}_{U} \rightarrow U$ be a conformal map such that $\varphi_{U}(O)=\infty$. We always assume that $\varphi_{U}$ is extended over $\mathcal{A}_{U}$; however, this is in general not continuous.

We say that $x \in X$ is accessible from $U$ if there exists an angle $\alpha \in[0,2 \pi)$ such that the (conformal) external ray $R_{\alpha}$ lands on $x$. It is well-known that a point $x \in X$ is accessible from $U$ if and only if there exists a continuum $Y \subset \bar{U}$ such that $Y \cap X=\{x\}$. Moreover, in this case $\overline{\varphi_{U}^{-1}(Y \backslash\{x\})} \cap S_{U}^{1}=\{\alpha\}$ is a single point and $R_{\alpha}$ lands on $x$ in $X$; see Milnor [14, Corollary 17.10]. 
We will need the following well-known facts from Carathéodory theory; see for instance [14] and [17. Suppose $U \subset \mathbb{C}^{*}$ is a simply connected open set containing $\infty$. A crosscut $C$ of $U$ is an open arc in $U \backslash\{\infty\}$ whose closure is a closed arc with its endpoints in $\partial U$. If $C$ is a crosscut, denote its shadow by $\operatorname{Sh}(C)$, defined as the component of $U \backslash C$ disjoint from $\{\infty\}$. It is known that if $C$ is a crosscut of $U$, then $\varphi_{U}^{-1}(C)$ is a crosscut of $\mathbb{D}_{U}$ and $\varphi_{U}^{-1}(\operatorname{Sh}(C))=\operatorname{Sh}\left(\varphi_{U}^{-1}(C)\right)$, where the shadow of a crosscut $D$ in $\mathbb{D}_{U}$ is defined as the component of $\mathbb{D}_{U} \backslash D$ disjoint from $O$. A sequence of crosscuts $\left\{C_{i}\right\}$ is a fundamental chain provided that $C_{i+1} \subset \operatorname{Sh}\left(C_{i}\right)$ and $\overline{C_{i+1}} \cap \overline{C_{i}}=\emptyset$ for each $i$, and $\lim \operatorname{diam} C_{i}=0$. Two fundamental chains $\left\{C_{i}\right\}$ and $\left\{C_{i}^{\prime}\right\}$ are equivalent if $\operatorname{Sh}\left(C_{n}\right)$ contains all but finitely many crosscuts $C_{i}^{\prime}$ and vice versa. An equivalence class of fundamental chains is called a prime end of $U$.

Given a fundamental chain $\left\{C_{i}\right\}$, the set $\lim \varphi_{U}^{-1}\left(C_{i}\right)$ is an angle $\alpha \in S_{U}^{1}$, to which we can identify the corresponding prime end. For a prime end $\alpha$ and a corresponding fundamental chain $\left\{C_{i}\right\}$ we can define the impression of $\alpha$ by $\operatorname{Imp}(\alpha):=\bigcap \overline{\operatorname{Sh}\left(C_{i}\right)}$. Since it is known that $\operatorname{Imp}(\alpha)$ does not depend on the choice of a fundamental chain, this is well-defined. The principal set of $R_{\alpha}$ or $\alpha$ is given by $\Pi(\alpha):=\overline{R_{\alpha}} \backslash R_{\alpha}$. It is known that $\Pi(\alpha) \subset \operatorname{Imp}(\alpha)$ and that for each point $x \in \Pi(\alpha)$ there exists a fundamental chain $\left\{C_{i}\right\}$ of the prime end $\alpha$ such that $\lim C_{i}=x$. Such a family $\left\{C_{i}\right\}$ is called a defining family of crosscuts of the prime end $\alpha$.

The main tool we will be using in this paper is a modification of a partition of $U$ which is due to Kulkarni and Pinkall [12]; see [5, 15, 4, for applications (of the modified version). Let $\mathcal{B}$ denote the collection of all maximal round open balls $B(z, r) \subset U$ (that is, open balls in the sphere equipped with the spherical metric, of radius $r$ about $z$ and such that $|\partial(B(z, r)) \cap \partial U| \geq 2)$. Let $\mathcal{C}$ be the collection of all centers of such balls and for $c \in \mathcal{C}$ let $r(c)$ be the corresponding radius. Note that for each $c \in \mathcal{C}$, the ball $B:=B(c, r(c))$ is conformally equivalent to the unit disk $\mathbb{D}$ and hence can be endowed with the hyperbolic metric, denoted by $\rho_{B}$. Geodesics in this metric are intersections of $B$ with round circles $C \subset \mathbb{C}^{*}$ which cross the boundary $\partial B$ perpendicularly. For every geodesic $g$, there are exactly two components of $\bar{B} \backslash \bar{g}$. The closure in $\bar{B}$ of such a component is a hyperbolic halfplane of $B$. Let $F(c)$ denote the hyperbolic convex hull of $\overline{B(c, r(c))} \cap \partial U$ in $\overline{B(c, r(c))}$, where $B(c, r(c))$ is equipped with $\rho_{B(c, r(c))}$, given by the intersection of all hyperbolic halfplanes of $B(c, r(c))$ that contain $\overline{B(c, r(c))} \cap \partial U$; see Figure 1 . Denote $\mathcal{F}:=\{F(c): c \in \mathcal{C}\}$. The following theorem is due to Kulkarni and Pinkall; see [12]:

Theorem 2.1 (Kulkarni-Pinkall). For each $z \in U$ there exists a unique $c \in \mathcal{C}$ such that $z \in F(c)$. In particular, if $c_{1} \neq c_{2} \in \mathcal{C}$, then $F\left(c_{1}\right) \cap F\left(c_{2}\right) \cap U=\emptyset$.

Hence $\mathcal{F}^{\prime}:=\{F(c) \cap U: c \in \mathcal{C}\}$ is a partition of $U$ into disjoint closed subsets. Let $\mathcal{K P}(c)$ be the collection of all nondegenerate chords in $\partial F(c)$ and observe that their endpoints are accessible points in $\partial U$. Consider the collection $\mathcal{K} \mathcal{P}:=\bigcup_{c \in \mathcal{C}} \mathcal{K P}(c)$. Note that for $a, b \in \partial U$ there may be uncountably many chords in $\mathcal{K} \mathcal{P}$ joining $a$ and $b$. We will replace the collection $\mathcal{K} \mathcal{P}$ by the collection $\mathcal{H}$, defined below, which has the property that for each two accessible points in $\partial U$ there is at most one chord joining them. We denote by $G(\alpha, \beta)$ the hyperbolic geodesic in $\mathbb{D}_{U}$ connecting $\alpha, \beta \in S_{U}^{1}$. The collection

$$
\mathcal{L}:=\left\{G(\alpha, \beta): \exists g \in \mathcal{K} \mathcal{P}, \overline{\varphi_{U}^{-1}(g)} \text { has endpoints } \alpha, \beta \in S_{U}^{1}\right\}
$$




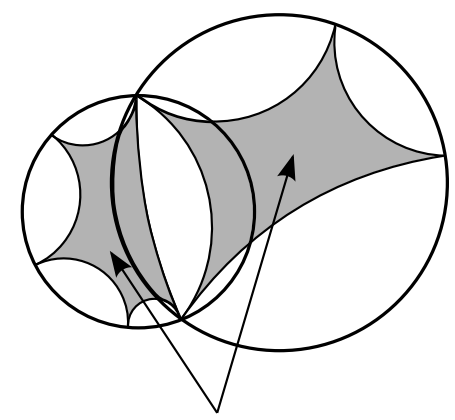

Figure 1. The grey areas depict two hyperbolic convex hulls.

is a lamination of $\mathbb{D}_{U}$ in the sense of Thurston, [19]. We modify the partition of $U$ by, instead of $\mathcal{K} \mathcal{P}$, considering the collection

$$
\mathcal{H}:=\left\{\varphi_{U}(G(\alpha, \beta)): G(\alpha, \beta) \in \mathcal{L}\right\} .
$$

We call its elements hyperbolic leaves and a component of $U \backslash \bigcup \mathcal{H}$ a hyperbolic gap. By Fokkink et al. [5] we have that for every hyperbolic leaf $\ell$ there is a $c \in \mathcal{C}$ such that $\ell \subset B(c, r(c))$; however, this correspondence is no longer unique. Nevertheless, this can be strengthened to stating that the collection of hyperbolic leaves and hyperbolic gaps has essentially the same property as $\mathcal{F}$ as given in Theorem 2.1.

Theorem 2.2 ([5, Theorem 4.5]). For each $z \in U$ there exists either a unique hyperbolic leaf $\ell \in \mathcal{H}$ so that $z \in \ell$, or a unique hyperbolic gap $\Gamma$ generated by $\mathcal{H}$ so that $z \in \Gamma$. In particular, any two distinct hyperbolic leaves do not cross each other in $U$.

A part of the correspondence between balls and elements of the lamination $\mathcal{K P}$ remains valid for the hyperbolic lamination $\mathcal{H}$; see [5, Lemma 4.1]:

Lemma 2.3 (Jörgensen). Let $B \subset U$ be a round open ball in $\mathbb{C}^{*}$. Let $G \subset \mathbb{D}_{U}$ be a hyperbolic geodesic. Then $\varphi_{U}(G) \cap B$ is connected. Moreover, if $\ell \in \mathcal{H}$ and $\bar{\ell} \backslash \ell \subset \partial B \cap \partial U$, then $\ell \subset B$.

It follows from Lemma 2.3 (see [5, Lemma 4.4]) that for each hyperbolic leaf or gap $F$, there exists $B \in \mathcal{B}$ such that $\bar{F} \subset \bar{B}$. For completeness, we include a useful corollary following [5, Proposition 5.1].

Corollary 2.4. Suppose that $\left\{\ell_{i}\right\} \subset \mathcal{H}$ and suppose there are $x_{i} \in \ell_{i}$ such that $x_{i} \rightarrow x$ for some $x \in U$. Then there is a unique hyperbolic leaf $\ell \in \mathcal{H}$ that contains $x$ and, moreover, $\lim \overline{\ell_{i}}=\bar{\ell}$. If $\left\{\ell_{i}\right\}$ is a convergent sequence in $\mathcal{H}$, but there is no convergent sequence $\left\{x_{i}\right\}$ with $x_{i} \in \ell_{i}$ and $\lim x_{i} \in U$, then $\lim \overline{\ell_{i}}$ is a point in $\partial U$.

We will also use the following well-known theorem (e.g. [17, Theorem 4.20]).

Theorem 2.5 (Gehring-Hayman Theorem). There exists a universal constant $K$ such that for any conformal map $\varphi: \mathbb{D} \rightarrow \mathbb{C}$, if $z_{1}, z_{2} \in \overline{\mathbb{D}}, J$ is an arc in $\mathbb{D}$ from $z_{1}$ to $z_{2}$, and $G$ is the hyperbolic geodesic from $z_{1}$ to $z_{2}$, then $\operatorname{diam} \varphi(G) \leq K \operatorname{diam} \varphi(J)$.

The circular order $<$ on $S^{1}$ is defined as follows. For $\alpha \neq \beta \neq \gamma \in S^{1}$ we have $\alpha<\beta<\gamma$, whenever following the arc from $\alpha$ along $S^{1}$ in the direction of increasing arguments and stopping when $\gamma$ is encountered for the first time, one encounters $\beta$ before $\gamma$. 
Definition 2.6. We say that a homeomorphism $\hat{h}: S^{1} \rightarrow S^{1}$ preserves the circular order on $S^{1}$ if $\hat{h}(\alpha)<\hat{h}(\beta)<\hat{h}(\gamma)$ whenever $\alpha<\beta<\gamma$ in the circular order on $S^{1}$.

\section{EXTENDING A HOMEOMORPHISM OVER ONE COMPLEMENTARY DOMAIN}

Throughout this section we will assume that we are given a homeomorphism between two continua $h: X \rightarrow Y$ in the sphere. We may also assume that $U$ and $V$ are corresponding complementary domains both containing $\infty$.

Definition 3.1. A function $f: S_{U}^{1} \rightarrow S_{V}^{1}$ is called $M$-uniform if $f\left(\mathcal{A}_{U}\right) \subset \mathcal{A}_{V}$ and $f \uparrow \mathcal{A}_{U}$ is uniformly continuous with respect to the Mazurkiewicz metrics of $\mathcal{A}_{U}$ and $\mathcal{A}_{V}$.

Remark 3.2. Observe that if $h \uparrow \partial U: \partial U \rightarrow \partial V$ induces a homeomorphism $\hat{h}$ : $S_{U}^{1} \rightarrow S_{V}^{1}$ and if there exists a homeomorphic extension $H: X \cup U \rightarrow Y \cup V$ of $h \uparrow \partial U$, then $\hat{h}$ is M-uniform.

Given the proper simply connected subset $U \subset \mathbb{C}^{*}$ we construct the lamination $\mathcal{L}$ of $\mathbb{D}_{U}$ as described in the preliminaries and denote it by $\mathcal{L}_{U}$, and the corresponding collection of hyperbolic leaves $\mathcal{H}$, denoted by $\mathcal{H}_{U}:=\varphi_{U}\left(\mathcal{L}_{U}\right)$ in $U$.

Let $U$ and $V$ be the corresponding domains of $X$ and $Y$. On the two copies of the hyperbolic disk, we now change to the Cayley-Klein model. Therefore consider the homeomorphism $g: \overline{\mathbb{D}} \rightarrow \overline{\mathbb{D}}$ from the Poincaré model to the Cayley-Klein model, given in polar coordinates by $g(r, \theta)=\left(2 r /\left(1+r^{2}\right), \theta\right)$. This is the identity on the boundary $S^{1}$ of $\mathbb{D}$, the map $g$ preserves radial line segments and for any two points $\alpha_{1}, \alpha_{2} \in S^{1}$ the hyperbolic geodesic $G\left(\alpha_{1}, \alpha_{2}\right)$ joining $\alpha_{1}$ and $\alpha_{2}$ is mapped to the straight line segment $\alpha_{1} \alpha_{2}$, which is the (open) Euclidean chord of the unit disk from $\alpha_{1}$ to $\alpha_{2}$.

Lemma 3.3. Suppose there exists a homeomorphism $\hat{h}: S_{U}^{1} \rightarrow S_{V}^{1}$ induced by $h\left\lceil\partial U: \partial U \rightarrow \partial V\right.$. Then there exists a homeomorphic extension $\hat{H}: \overline{\mathbb{D}}_{U} \rightarrow \overline{\mathbb{D}}_{V}$ of $\hat{h}$ such that for every hyperbolic geodesic $G(\alpha, \beta) \in \mathcal{L}_{U}$ we have that $\hat{H}(G(\alpha, \beta))$ is the hyperbolic geodesic joining $\hat{h}(\alpha)$ and $\hat{h}(\beta)$. In particular $\mathcal{L}_{V}:=\{\hat{H}(G(\alpha, \beta))$ : $\left.G(\alpha, \beta) \in \mathcal{L}_{U}\right\}$ forms a lamination in $\mathbb{D}_{V}$.

Proof. Define $\mathcal{E}_{U}:=g\left(\mathcal{L}_{U}\right)$, a Euclidean lamination of the unit disk consisting of Euclidean chords. Denote the Euclidean chord from $\alpha \in S^{1}$ to $\beta \in S^{1}$ in $\mathbb{D}$ by $\alpha \beta$. Define $\mathcal{E}_{V}:=\left\{\hat{h}(\alpha) \hat{h}(\beta): \alpha \beta \in \mathcal{E}_{U}\right\}$ and observe that this is a lamination as well, since $\hat{h}$ is a homeomorphism. We will first define a map $L: \overline{\mathbb{D}}_{U} \rightarrow \overline{\mathbb{D}}_{V}$. For every $\alpha \beta \in \mathcal{E}_{U}$, let $L \uparrow \overline{\alpha \beta}: \overline{\alpha \beta} \rightarrow \overline{\hat{h}(\alpha) \hat{h}(\beta)}$ be the linear extension of $\hat{h} \uparrow\{\alpha, \beta\}$. If $\Gamma$ is a gap in $\mathcal{E}_{U}$, that is, a component of $\mathbb{D}_{U} \backslash \cup \mathcal{E}_{U}$, denote its (Euclidean) barycenter by $b_{\Gamma}$. Note that $L$ is already defined on the entire boundary of $\Gamma$. Then the image of the union of all the leaves in the boundary of $\Gamma$ forms the boundary of a gap $\Delta$ of $\mathcal{E}_{V}$. Let $b_{\Delta}$ denote the barycenter of $\Delta$.

Define $L\left(b_{\Gamma}\right)=b_{\Delta}$ and extend $L$ over $\Gamma$ by mapping, for each $x \in \partial \Gamma$, the segment $x b_{\Gamma}$ linearly onto the segment $L(x) b_{\Delta}$. Then $L: \overline{\mathbb{D}}_{U} \rightarrow \overline{\mathbb{D}}_{V}$ is a homeomorphism which maps every leaf $\ell \in \mathcal{E}_{U}$ linearly onto the corresponding leaf in $\mathcal{E}_{V}$. Finally define $\hat{H}: \overline{\mathbb{D}}_{U} \rightarrow \overline{\mathbb{D}}_{V}$ by $\hat{H}:=g^{-1} \circ L \circ g$. Then $\hat{H}$ is the required homeomorphism. 
Theorem 3.4. Suppose there exists a homeomorphism $\hat{h}: S_{U}^{1} \rightarrow S_{V}^{1}$ induced by $h \uparrow \partial U: \partial U \rightarrow \partial V$, which is $M$-uniform. Then there exists a homeomorphic extension $H: X \cup U \rightarrow Y \cup V$ of $h$.

Proof. Apply Lemma 3.3 to find an extension $\hat{H}: \overline{\mathbb{D}}_{U} \rightarrow \overline{\mathbb{D}}_{V}$ of $\hat{h}$ and define $H: X \cup U \rightarrow Y \cup V$ to be $H \uparrow U:=\varphi_{V} \circ \hat{H} \uparrow \mathbb{D}_{U} \circ \varphi_{U}^{-1}$ and $H \uparrow X:=h$. This is clearly a well-defined bijective extension of $h$. Denote the induced lamination for $V$ by $\mathcal{H}_{V}:=H\left(\mathcal{H}_{U}\right)=\varphi_{V}\left(\mathcal{L}_{V}\right)$. By $T$ we denote a hyperbolic object that is either a hyperbolic leaf or a hyperbolic gap for $\mathcal{H}_{U}$. By the above considerations and compactness of $X \cup U \subset \mathbb{C}^{*}$, we only need to verify continuity of $H$. By construction it is clear that $H \uparrow U: U \rightarrow V$ is a homeomorphism. This also holds for $H \uparrow \bar{T}$, with $T$ a hyperbolic object (see [4]). Hence we only have to be concerned about sequences in $U$ lying in infinitely many distinct hyperbolic objects that converge to a point in $\partial U$. Without loss of generality, we can consider a convergent sequence $\left\{x_{n}\right\} \subset U$ with $x_{n} \in T_{n}$ and $T_{n} \neq T_{m}$ whenever $n \neq m$, for which $x:=\lim x_{n} \in \partial U$. There are two cases: either diam $T_{n} \rightarrow 0$ or diam $T_{n} \nrightarrow \rightarrow 0$. We will show that in either case $H\left(x_{n}\right) \rightarrow H(x)$.

Case one. Suppose that diam $T_{n} \rightarrow 0$. For each $n$ we can find two accessible angles $\alpha_{n}, \beta_{n}$ with $x_{\alpha_{n}}$ and $x_{\beta_{n}}$ in $\bar{T}_{n} \cap \partial U$ that can be connected by an arc whose image runs in $\overline{T_{n}} \backslash \partial U$. Hence $\rho_{U}\left(\alpha_{n}, \beta_{n}\right) \rightarrow 0$ and by the assumption of Muniformity we have that $\rho_{V}\left(\hat{h}\left(\alpha_{n}\right), \hat{h}\left(\beta_{n}\right)\right) \rightarrow 0$. This implies that there have to be $\operatorname{arcs}$ in $\mathbb{D}_{V}$ with small images in $V$ connecting $h\left(x_{\alpha_{n}}\right)$ and $h\left(x_{\beta_{n}}\right)$, independent of the choice of $\alpha_{n}, \beta_{n}$. In addition, by construction and Theorem 2.5 we find that for every choice of $\ell_{n} \subset \partial T_{n}$ we have diam $H\left(\ell_{n}\right) \rightarrow 0$. Note that $\partial H\left(T_{n}\right)=H\left(\partial T_{n}\right)$ (see [4). We conclude that diam $H\left(T_{n}\right) \rightarrow 0$. Furthermore, in particular we find that if $\lim x_{\alpha_{n}}=x$, then $h(x)=\lim h\left(x_{\alpha_{n}}\right)=\lim H\left(T_{n}\right)=\lim H\left(x_{n}\right) \in \partial V$ and $H\left(x_{n}\right) \rightarrow H(x)=h(x)$.

Case two. Suppose that diam $T_{n} \nrightarrow \neg 0$. By Corollary 2.4. we may assume that $\lim T_{n}=\bar{\ell}$ for some hyperbolic leaf $\ell \in \mathcal{H}_{U}$. Moreover, we may assume that the sequence $T_{n}$ converges monotonically to $\ell$ from one side of $\ell$. Denote the endpoints of $\varphi_{U}^{-1}(\ell)$ by $\alpha$ and $\beta$. It follows that $\lim \sup H\left(T_{n}\right) \subset \operatorname{Imp}(\hat{h}(\alpha)) \cup H(\ell) \cup \operatorname{Imp}(\hat{h}(\beta))$. We will first show that $\lim H\left(T_{n}\right)=H(\bar{\ell})$. Let $B_{n} \in \mathcal{B}$ such that $T_{n} \subset B_{n}$. Then $B_{n} \rightarrow B \in \mathcal{B}$ and $\ell \subset B$.

Now construct disjoint $\operatorname{arcs} \varphi_{V}\left(\hat{L}^{\prime}\right)\left(\varphi_{V}\left(\hat{R}^{\prime}\right)\right)$ with endpoint $h(a)(h(b))$, for $\varphi_{U}(\alpha)=a\left(\varphi_{U}(\beta)=b\right.$, resp. $)$ such that $H\left(T_{n}\right)$ is located "between" these two arcs. To see how the arcs $\hat{L}^{\prime}$ and $\hat{R}^{\prime}$ are constructed note first that the sets of centers $\mathcal{C}$ of maximal balls is an $\mathbb{R}$-tree [2, 6. In particular it is uniquely arcwise connected. Let $\left[c_{1}, c\right] \subset \mathcal{C}$ denote the unique arc whose endpoints are the centers of $B_{1}$ and $B$. Let $r$ be the radius of $B, \delta$ the diameter of $\ell$ and let $\eta=\min \{r, \delta\}$. We may assume that $d_{H}\left(\bigcup T_{n}, \ell\right)<\eta / 10$ and $c_{n} \in\left[c_{1}, c\right] \subset B(c, \eta / 10)$ for each $n$, where $c_{n}$ is the center of $B_{n}$. Let $L$ be the union of all leaves in $\mathcal{H}_{U}$ such that both endpoints are contained $\overline{B^{\prime}} \cap X \cap B(a, \eta / 5)$ for some $B^{\prime} \in \mathcal{B}$ with center $c^{\prime} \in\left[c_{1}, c\right]$ together with all points of $\bigcup_{c^{\prime} \in\left[c_{1}, c\right]} \overline{B\left(c^{\prime}, r\left(c^{\prime}\right)\right)} \cap X \cap B(a, \eta / 5)$ (see [5, Lemma 6.1.1]). Note that for each $n, T_{n} \cap L$ is connected. Construct $R$ similarly. Observe that for each $n, T_{n}$ is located "between" $L$ and $R$.

Then $\hat{H} \circ \varphi_{U}^{-1}(L)=\hat{L}$ and $\hat{H} \circ \varphi_{U}^{-1}(R)=\hat{R}$ are arcs in $\mathbb{D}_{V}$. We can replace the closure of every component of $\hat{L} \backslash \hat{H} \circ \varphi_{U}^{-1}\left(T_{n}\right)$ by an arc with the same endpoints which meets $\partial \mathbb{D}_{V}$ nontangentially at both endpoints and is otherwise disjoint from 
$\partial \mathbb{D}_{V}$. Denote the new arc by $\hat{L}^{\prime}$ and similarly construct $\hat{R}^{\prime}$. By the Lindelöf Theorem, 17], $\varphi_{V}\left(\hat{L}^{\prime}\right)$ and $\varphi_{V}\left(\hat{R}^{\prime}\right)$ are $\operatorname{arcs}$ in $\bar{V}$ with endpoints $h(a)$ and $h(b)$ such that $H\left(T_{n}\right)$ is located "between them". Hence $\lim H\left(T_{n}\right) \subset H(\bar{\ell})$, as desired.

We may assume that $\lim x_{n}=x=\varphi_{U}(\alpha)$. Suppose $\lim H\left(x_{n}\right) \neq H\left(\varphi_{U}(\alpha)\right)$ and we may assume that $\lim H\left(x_{n}\right)=z \in H(\bar{\ell}) \backslash\left\{H\left(\varphi_{U}(\alpha)\right)\right\}$. Then $\lim \varphi_{V}^{-1}\left(H\left(x_{n}\right)\right) \in$ $\varphi_{V}^{-1}(H(\bar{\ell})) \backslash \varphi_{V}^{-1}\left(H\left(\varphi_{U}(\alpha)\right)\right)=\hat{H}\left(\varphi_{U}^{-1}(\bar{\ell})\right) \backslash\{\hat{h}(\alpha)\}$. However, $x_{n} \rightarrow \varphi_{U}(\alpha)$ implies $\varphi_{U}^{-1}\left(x_{n}\right) \rightarrow \alpha$ and hence $\varphi_{V}^{-1}\left(H\left(x_{n}\right)\right)=\hat{H}\left(\varphi_{U}^{-1}\left(x_{n}\right)\right) \rightarrow \hat{h}(\alpha)$. This contradiction completes the proof.

We have the following corollaries.

Corollary 3.5. Let $X$ and $Y$ be nonseparating planar continua and suppose there exists a homeomorphism $h: X \rightarrow Y$. Suppose there exists a homeomorphism $\hat{h}: S_{U}^{1} \rightarrow S_{V}^{1}$ induced by $h\lceil\partial U: \partial U \rightarrow \partial V$ which preserves the circular order and is M-uniform. Then $h$ is isotopic to the identity on $X$.

Proof. We may view $X$ and $Y$ as subsets of the sphere and apply Theorem 3.4 to find an extension $H$ of $h$ such that $H: \mathbb{C}^{*} \rightarrow \mathbb{C}^{*}$ is a homeomorphism. Then we can compose $H$ by an orientation-preserving homeomorphism $K: \mathbb{C}^{*} \rightarrow \mathbb{C}^{*}$ such that $K \uparrow Y=\operatorname{id}_{Y}$ and $K(H(\infty))=\infty$. Hence we obtain a homeomorphism $G=K \circ H$ which extends $h$ and fixes the point at infinity, so that its restriction to $\mathbb{C}$ is a homeomorphism as well and an extension of $h$. Since $\hat{h}$ preserves the circular order, $H$ and thus $G$ is orientation preserving. Hence, $h$ is isotopic to the identity [10, Theorem 6.4].

Corollary 3.6. Suppose there exists a homeomorphism $\hat{h}: S_{U}^{1} \rightarrow S_{V}^{1}$ induced by $h \uparrow \partial U: \partial U \rightarrow \partial V$ which is M-uniform. Then for every $\alpha \in S_{U}^{1}$ we have $h(\Pi(\alpha))=\Pi(\hat{h}(\alpha))$, the inverse $\hat{h}^{-1}$ is $M$-uniform as well and $\hat{h}: S_{U}^{1} \rightarrow S_{V}^{1}$ is a homeomorphism with respect to both the usual and the Mazurkiewicz metrics on the entire prime end circles as defined in Section 5 .

\section{EXTENDing A HOMEOMORPHISM OVER MORE THAN ONE COMPLEMENTARY DOMAIN}

As in the previous section we will assume that we are given a homeomorphism between two continua $h: X \rightarrow Y$ in the sphere.

Definition 4.1. Suppose that $U_{n}$ and $V_{n}$ are corresponding domains, $\hat{h}_{n}: S_{U_{n}}^{1} \rightarrow$ $S_{V_{n}}^{1}$ are homeomorphisms and $\rho_{U_{n}}$ and $\rho_{V_{n}}$ are Mazurkiewicz metrics on $\mathcal{A}_{U_{n}}$ and $\mathcal{A}_{V_{n}}$. Then we say that the family $\left\{\hat{h}_{n}: S_{U_{n}}^{1} \rightarrow S_{V_{n}}^{1}\right\}$ is uniformly equicontinuous in the Mazurkiewicz metric provided that

$$
\forall \varepsilon \exists \delta \forall n \forall \alpha, \beta \in \mathcal{A}_{U_{n}} \quad\left(\rho_{U_{n}}(\alpha, \beta)<\delta \Rightarrow \rho_{V_{n}}\left(\hat{h}_{n}(\alpha), \hat{h}_{n}(\beta)\right)<\varepsilon\right) .
$$

Remark 4.2. Observe that if $h \uparrow \partial U_{n}: \partial U_{n} \rightarrow \partial V_{n}$ induces a homeomorphism $\hat{h}_{n}: S_{U_{n}}^{1} \rightarrow S_{V_{n}}^{1}$ for every $n$ and if there exists a homeomorphic extension $H$ on $X \cup \bigcup U_{n}$ of $h$, then the family $\left\{\hat{h}_{n}\right\}$ is uniformly equicontinuous in the Mazurkiewicz metric.

Theorem 4.3. Let $X$ and $Y$ be two continua in the sphere and suppose there exists a homeomorphism $h: X \rightarrow Y$. Suppose that there exists a bijection $\psi$ between the complementary components of $X$ and $Y$ such that whenever $U_{n}$ is a component of 
$\mathbb{C}^{*} \backslash X$ and $V_{n}=\psi\left(U_{n}\right)$, then $h\left\lceil\partial U_{n}: \partial U_{n} \rightarrow \partial V_{n}\right.$ induces a homeomorphism $\hat{h}_{n}: S_{U_{n}}^{1} \rightarrow S_{V_{n}}^{1}$ between the circles of prime ends of $U_{n}$ and $V_{n}$, respectively. Furthermore, assume that the family of the maps $\left\{\hat{h}_{n}\right\}$ is uniformly equicontinuous in the Mazurkiewicz metric.

Then $h$ can be extended to a homeomorphism $H: \mathbb{C}^{*} \rightarrow \mathbb{C}^{*}$ of the entire sphere.

Proof. Enumerate the complementary domains of $X$ as $U_{1}, U_{2}, \ldots$ and for each $n \geq 1$, let $V_{n}=\psi\left(U_{n}\right)$ be the complementary domain of $Y$ which corresponds to $U_{n}$. Apply Lemma 3.3 to find an extension $\hat{H}_{n}: \overline{\mathbb{D}}_{U_{n}} \rightarrow \overline{\mathbb{D}}_{V_{n}}$ of the homeomorphism $\hat{h}_{n}: S_{U_{n}}^{1} \rightarrow S_{V_{n}}^{1}$. Analogously to Theorem 3.4, put $H_{n}: X \cup U_{n} \rightarrow Y \cup V_{n}$ to be $H_{n} \uparrow U_{n}:=\varphi_{V_{n}} \circ \hat{H} \uparrow \mathbb{D}_{U_{n}} \circ \varphi_{U_{n}}^{-1}$ and $H_{n} \uparrow X:=h$. Observe that composing by Möbius transformations we can treat all complementary domains in a similar fashion. It is clear that each $H_{n}$ is a homeomorphism. Put $H=\bigcup H_{n}$. If $X$ has only finitely many complementary domains we are done. If not, it remains to be established whether $H$ is continuous on a sequence $\left\{x_{i}\right\} \rightarrow x \in X$ with $x_{i} \in U_{n_{i}}$ and $n_{i} \neq n_{j}$ whenever $i \neq j$. Let $T_{n_{i}}$ denote the hyperbolic object (either a leaf or a gap) generated by the lamination $\mathcal{H}_{U_{n_{i}}}$ of $U_{n_{i}}$ for which $x_{i} \in T_{n_{i}}$. By Lemma 2.3. there exists a maximal ball (though possibly not unique) $B_{n} \subset U_{n_{i}}$ with $T_{n_{i}} \subset B_{n}$. Clearly, diam $B_{n} \rightarrow 0$; hence also diam $T_{n_{i}} \rightarrow 0$. Thus we may replace our sequence $\left\{x_{i}\right\}$ by a sequence $\left\{x_{i}^{\prime}\right\}$, where $x_{i}^{\prime} \in \overline{\ell_{i}} \subset \partial T_{n_{i}}$. Now since diam $\ell_{i} \rightarrow 0$, we have for the angles $\alpha_{i}, \beta_{i}$ corresponding to the endpoints of $\ell_{i}$ that $\rho_{U_{n_{i}}}\left(\alpha_{i}, \beta_{i}\right) \rightarrow 0$ and hence, by the assumption, $\rho_{V_{n_{i}}}\left(\hat{h}_{n_{i}}\left(\alpha_{i}\right), \hat{h}_{n_{i}}\left(\beta_{i}\right)\right) \rightarrow 0$. By construction and Theorem 2.5 we have that diam $H\left(\ell_{i}\right) \rightarrow 0$; hence $H\left(\lim x_{i}\right)=H\left(\lim x_{i}^{\prime}\right)=H(x)$.

In fact, we now obtain a homeomorphism $H: \mathbb{C}^{*} \rightarrow H(X) \subset \mathbb{C}^{*}$; hence $H(X)=$ $\mathbb{C}^{*}$.

We have the following corollaries.

Corollary 4.4. Let $X$ and $Y$ be two continua in the sphere and suppose there exists a homeomorphism $h: X \rightarrow Y$. Suppose that there exists a bijection $\psi$ between the complementary components of $X$ and $Y$ such that whenever $U_{n}$ is a component of $\mathbb{C}^{*} \backslash X$ and $V_{n}=\psi\left(U_{n}\right)$, then $h \uparrow \partial U_{n}: \partial U_{n} \rightarrow \partial V_{n}$ induces a homeomorphism $\hat{h}_{n}: S_{U_{n}}^{1} \rightarrow S_{V_{n}}^{1}$ between the circles of prime ends of $U_{n}$ and $V_{n}$, respectively. In addition, assume there exists an $n_{0}$ for which $\hat{h}_{n_{0}}$ preserves the circular order. Furthermore, assume the family of the maps $\left\{\hat{h}_{n}\right\}$ is uniformly equicontinuous in the Mazurkiewicz metric. Then $h$ is isotopic to the identity.

Corollary 4.5. Let $X$ and $Y$ be locally connected continua in the sphere and suppose there exists a homeomorphism $h: X \rightarrow Y$. Suppose that there exists a bijection $\psi$ between the complementary components of $X$ and $Y$ such that whenever $U_{n}$ is a component of $\mathbb{C}^{*} \backslash X$ and $V_{n}=\psi\left(U_{n}\right)$, then $h \uparrow \partial U_{n}: \partial U_{n} \rightarrow \partial V_{n}$ induces a homeomorphism $\hat{h}_{n}: S_{U_{n}}^{1} \rightarrow S_{V_{n}}^{1}$ between the circles of prime ends of $U_{n}$ and $V_{n}$, respectively. In addition, assume there exists an $n_{0}$ so that $\hat{h}_{n_{0}}$ preserves the circular order. Then $h$ is isotopic to the identity on $X$.

Proof. By Carathéodory's Theorem (e.g. [14, Theorem 17.14]) if $X$ is locally connected and $U$ is a complementary domain of $X$, then $\varphi_{U}: \mathbb{D}_{U} \rightarrow U$ extends to a continuous map from $\overline{\mathbb{D}}_{U}$ onto $\bar{U}$. Hence, $\varphi_{U}$ is uniformly continuous. This implies that $\mathcal{A}_{U}=S_{U}^{1}$ and also that the usual topology on $S_{U}^{1}$ coincides with the topology 
generated by the Mazurkiewicz metric $\rho_{U}$. Moreover, if an induced homeomorphism $\hat{h}: S_{U}^{1} \rightarrow S_{V}^{1}$ exists, then by compactness $\hat{h}$ is M-uniform.

It is well-known that if $Y$ is a locally connected continuum in the sphere and $d_{n}=\operatorname{diam}\left(V_{n}\right)$, where $d_{n}=0$ if $V_{n}=\emptyset$, then $\lim d_{n}=0$. Hence, the condition of uniform equicontinuity in Corollary 4.4 is now automatically satisfied.

We claim that the condition of uniform equicontinuity in the Mazurkiewicz metric in Corollary 4.4 is necessary. For omitting the condition, we have a counterexample in the case with one complementary domain and in the case with infinitely many complementary domains. For this, we use the continua sketched in Figure 2.

Example 4.6. In Figure 2, consider the upper continuum $X$ and the lower continuum $Y$ sketched with solid and dashed lines and ignore the dotted lines. Both consist of curled hairs, converging to a vertical segment and connected to each other by a horizontal line, together with hairs emanating from the limit vertical segment and converging to that vertical segment as well. Now $X$ and $Y$ both have one complementary domain. Furthermore, they are homeomorphic; let $h: X \rightarrow Y$ denote the natural homeomorphism. Let $U=\mathbb{C}^{*} \backslash X$ and $V=\mathbb{C}^{*} \backslash Y$ and denote by $\varphi_{U}: \mathbb{D}_{U} \rightarrow U$ and $\varphi_{V}: \mathbb{D}_{V} \rightarrow V$ the Riemann maps. There is an angle $\alpha \in S_{U}^{1}$ for which a ray lands at the point $x_{\alpha}$, the endpoint of the limit vertical segment of the curled hairs. Without loss of generality, there is an angle $\beta_{1}^{-}>\alpha$ such that $\varphi_{U}\left(\beta_{1}^{-}\right)=x_{\beta_{1}}$ and observe that $\varphi_{U}$ extends over the positively oriented arc $\left[\alpha, \beta_{1}^{-}\right]$and that the image of this arc is the union of the vertical line containing $x_{\alpha}$ and the horizontal line containing $x_{\beta_{1}}$. Continue to find an angle $\gamma_{1}>\beta_{1}^{-}$with $\varphi_{U}\left(\gamma_{1}\right)=x_{\gamma_{1}}$ and observe that $\varphi_{U}$ extends over an $\operatorname{arc}\left[\beta_{1}^{-}, \gamma_{1}\right]$ corresponding to the first curled hair, approached from the left. Next, there is an angle $\beta_{1}^{+}>\gamma_{1}$ that goes with $x_{\beta_{1}}$ and an arc $\left[\gamma_{1}, \beta_{1}^{+}\right]$wrapping around the right side of the curled hair. Continuing in this fashion, we find angles with for each $i \geq 1$ the property $\alpha<\beta_{i}^{-}<\gamma_{i}<\beta_{i}^{+}<\beta_{i+1}^{-}<\gamma_{i+1}<\beta_{i+1}<\alpha$. Necessarily, the length of arcs $\left[\beta_{i}^{-}, \beta_{i+1}^{-}\right]$tends to zero. Starting at $\alpha$, but going in the opposite direction, we can find angles $\alpha=\alpha_{0}>\delta_{1}>\alpha_{1}>\delta_{2}>\alpha_{2} \ldots$, with $\varphi_{U}\left(\delta_{i}\right)=x_{\delta_{i}}$ and $\varphi_{U}\left(\alpha_{i}\right)=x_{\alpha}$ so that the image of the $\operatorname{arcs}\left[\alpha_{i-1}, \alpha_{i}\right]$ wraps around the hair with endpoints $x_{\delta_{1}}$ and $x_{\alpha}$ respectively. We find that $\left\{\delta_{i}\right\}$ and $\left\{\alpha_{i}\right\}$ converge to the same angle as $\left\{\gamma_{i}\right\}$; however, note that the ray that corresponds to this particular angle does not land.

Observe that for $Y$, we find that $\varphi_{V}$ behaves analogously. Here we can find an angle $\alpha^{\prime}=\alpha_{0}^{\prime}$ and sequences of angles $\left\{\beta_{i}^{\prime-}\right\},\left\{\beta_{i}^{\prime+}\right\},\left\{\gamma_{i}^{\prime}\right\},\left\{\delta_{i}^{\prime}\right\}$ and $\left\{\alpha_{i}^{\prime}\right\}$ so that for $i \geq 1$ we have $\varphi_{V}\left(\alpha^{\prime}\right)=y_{\alpha^{\prime}}, \varphi_{V}\left(\beta_{i}^{\prime-}\right)=y_{\beta_{i}^{\prime}}=\varphi_{V}\left(\beta_{i}^{\prime+}\right), \varphi_{V}\left(\gamma_{i}^{\prime}\right)=y_{\gamma_{i}^{\prime}}$, $\varphi_{V}\left(\delta_{i}^{\prime}\right)=y_{\delta_{i}^{\prime}}$ and $\varphi_{V}\left(\alpha_{i}\right)=y_{\alpha^{\prime}}$ and furthermore $\alpha^{\prime}<\beta_{i}^{\prime-}<\gamma_{i}^{\prime}<\beta_{i}^{\prime+}<\beta_{i+1}^{\prime-}<$ $\gamma_{i+1}^{\prime}<\beta_{i+1}^{\prime+}<\alpha_{i+1}^{\prime}<\delta_{i+1}^{\prime}<\alpha_{i}^{\prime}<\delta_{i}^{\prime}<\alpha^{\prime}$. We can now define $\hat{h}$, as follows. If $\eta \in\left[\beta_{i}^{-}, \gamma_{i}\right]$, let $\hat{h}(\eta)$ be the unique angle in $\left[\beta_{i}^{\prime-}, \gamma_{i}^{\prime}\right]$ for which $h\left(\varphi_{U}(\eta)\right)=\varphi_{V}(\hat{h}(\eta))$. Similarly, if $\eta \in\left[\gamma_{i}, \beta_{i}^{+}\right]$, let $\hat{h}(\eta) \in\left[\gamma_{i}^{\prime}, \beta_{i}^{\prime+}\right]$ with $h\left(\varphi_{U}(\eta)\right)=\varphi_{V}(\hat{h}(\eta))$. Intervals $\left[\alpha, \beta_{1}^{-}\right]$and $\left[\beta_{i}^{+}, \beta_{i+1}^{-}\right]$can also be mapped to $\left[\alpha^{\prime}, \beta_{1}^{\prime-}\right]$ respectively $\left[\beta_{i}^{\prime+}, \beta_{i+1}^{\prime+}\right]$ so that $h\left(\varphi_{U}(\eta)\right)=\varphi_{V}(\hat{h}(\eta))$ and similarly $\left[\alpha_{i}, \delta_{i}\right]$ and $\left[\delta_{i}, \alpha_{i-1}\right]$ can be mapped to $\left[\alpha_{i}^{\prime}, \delta_{i}^{\prime}\right]$ respectively $\left[\delta_{i}^{\prime}, \alpha_{i-1}^{\prime}\right]$ for $i \geq 1$. The function $\hat{h}$ is now well-defined and evidently continuous on $S_{U}^{1} \backslash\left\{\lim \alpha_{i}\right\}$. However, by definition we also have that whenever $\lim \eta_{n}=\lim \alpha_{i}$, then $\lim \hat{h}\left(\eta_{n}\right)=\lim \hat{h}\left(\alpha_{i}\right)=\lim \alpha_{i}^{\prime}$. We can extend $\hat{h}\left\lceil\mathcal{A}_{U}\right.$ to find a homeomorphism $\hat{h}: S_{U}^{1} \rightarrow S_{V}^{1}$ induced by $h$. Moreover, the 

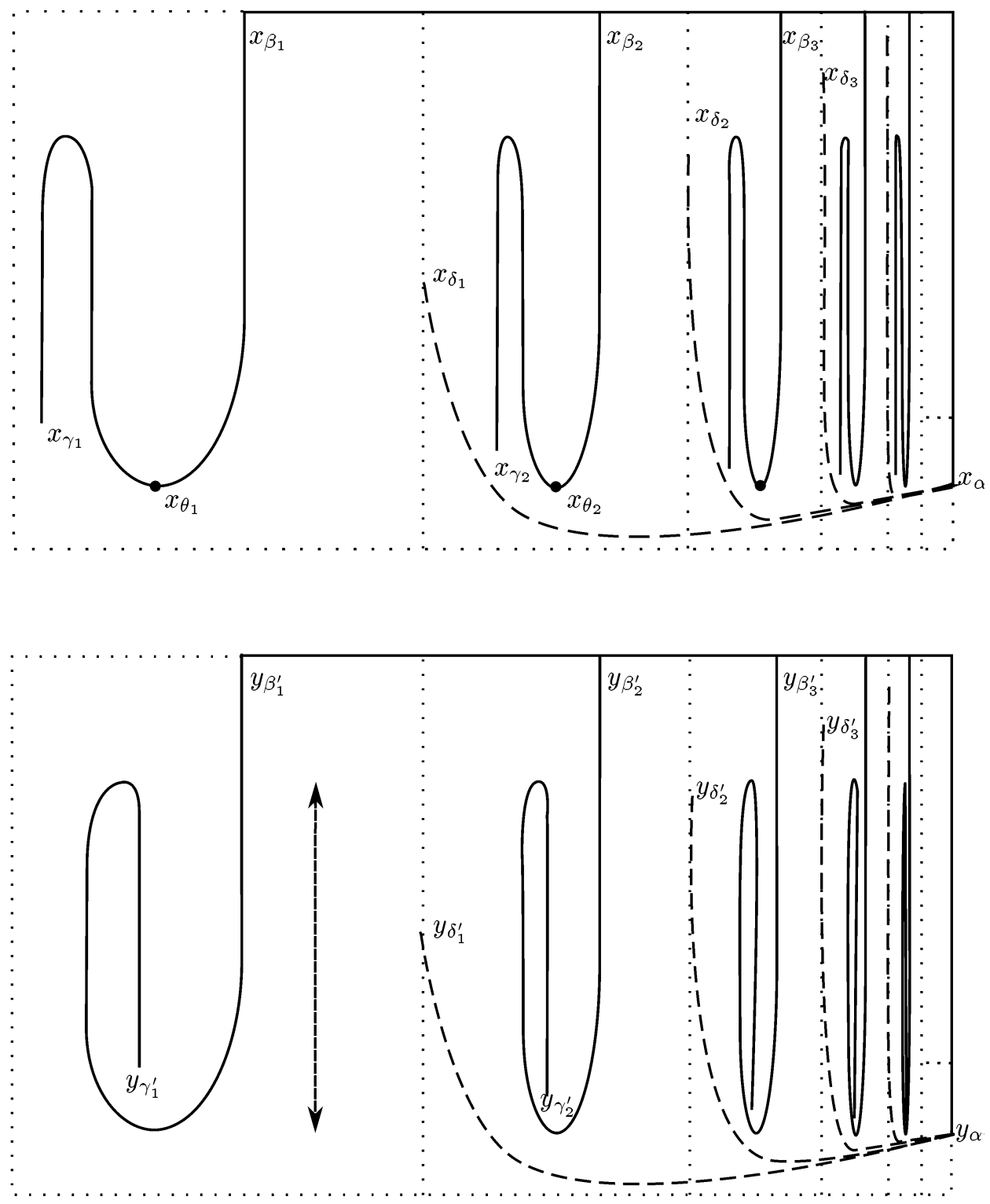

FiguRE 2. Homeomorphic continua that are not isotopic (one pair with solid and dashed lines and one pair with solid and dotted lines).

function $\hat{h} \uparrow \mathcal{A}_{U}$ is continuous with respect to the Mazurkiewicz metrics $\rho_{U}$ and $\rho_{V}$ because $\lim \alpha_{i} \notin \mathcal{A}_{U}$.

However, the function $\hat{h}$ is not M-uniform. Indeed, denote by $\theta_{i}$ the angle in $\left[\gamma_{i}, \beta_{i}^{+}\right]$for which $d\left(\varphi_{U}\left(\theta_{i}\right), x_{\beta_{i}}\right)$ is maximal. Let $\hat{h}\left(\theta_{i}\right)=\theta_{i}^{\prime}$ and recall that $\hat{h}\left(\gamma_{i}\right)=$ $\gamma_{i}^{\prime}$. Then $\rho_{U}\left(\gamma_{i}, \theta_{i}\right) \rightarrow 0$ while for the image points $\liminf \rho_{V}\left(\gamma_{i}^{\prime}, \theta_{i}^{\prime}\right)=\varepsilon>0$. Hence $h$ cannot be extended to a homeomorphism of the sphere. 
Observe that without the hairs with endpoints $x_{\delta_{i}}$ and $y_{\delta_{i}^{\prime}}$ the induced function $\hat{h} \uparrow \mathcal{A}_{U}$ would already have failed Mazurkiewicz continuity at the angle $\alpha$.

Example 4.7. Consider the upper continuum $X$ and the lower continuum $Y$ sketched in Figure 2 with solid and dotted lines, but not with dashed lines. Thus we have two homeomorphic continua that both have infinitely many complementary domains. Let $h$ denote the natural homeomorphism. Since the boundary of each complementary domain is locally connected, by the same argument as in Corollary 4.5, we can find homeomorphisms $\hat{h}_{n}: S_{U_{n}}^{1} \rightarrow S_{V_{n}}^{1}$, induced by $h\left\lceil\partial U_{n}\right.$ that are also homeomorphisms with respect to the Mazurkiewicz metrics. By compactness, we even have uniform continuity on each domain. However, the uniformity condition on all domains simultaneously fails. Suppose that $U_{i}$ is in the $i$ th rectangle. Then there are angles $\beta_{i}^{-}<\theta_{i}^{-}<\gamma_{i}<\theta_{i}^{+}<\beta_{i}^{+}$with $\varphi_{U_{i}}\left(\beta_{i}^{-}\right)=\varphi_{U_{i}}\left(\beta_{i}^{+}\right)=x_{\beta_{i}}, \varphi_{U_{i}}\left(\gamma_{i}\right)=x_{\gamma_{i}}$ and $\varphi_{U_{i}}\left(\theta_{i}^{+}\right)=\varphi_{U_{i}}\left(\theta_{i}^{-}\right)=x_{\theta_{i}}$. We can similarly find angles $\beta_{i}^{\prime-}, \beta_{i}^{\prime+}$ for $V_{i}$ and define $\hat{h}_{i}$ so that $\left[\beta_{i}^{-}, \beta_{i}^{+}\right]$is mapped onto $\left[\beta_{i}^{\prime-}, \beta_{i}^{\prime+}\right]$ with $h\left(\varphi_{U_{i}}(\eta)\right)=\varphi_{V_{i}}\left(\hat{h}_{i}(\eta)\right)$ for every $\eta \in S_{U_{i}}^{1}$. Then $\rho_{U_{i}}\left(\gamma_{i}, \theta_{i}^{-}\right) \rightarrow 0$, but $\rho_{V_{i}}\left(\hat{h}_{i}\left(\gamma_{i}\right), \hat{h}_{i}\left(\theta_{i}^{-}\right)\right) \nrightarrow \rightarrow 0$. Since the family $\left\{\hat{h}_{n}\right\}$ is not uniformly equicontinuous in the Mazurkiewicz metric, $h$ cannot be extended to a homeomorphism of the sphere.

\section{The Mazurkiewicz Metric}

We now consider a proper, simply connected open subset $U$ and the conformal map $\varphi_{U}: \mathbb{D}_{U} \rightarrow U$ as discussed in the preliminaries. We will identify the (entire) unit circle $S_{U}^{1}=\partial \mathbb{D}_{U}$ with the circle of prime ends of $U$ and equip it with the Mazurkiewicz metric $\rho_{U}$ defined as follows: if $\alpha=\beta$, set $\rho_{U}(\alpha, \beta)=0$; if $\alpha \neq \beta$, then

$$
\begin{aligned}
\rho_{U}(\alpha, \beta)=\inf \left\{\operatorname{diam} \varphi_{U}(J): J\right. & \text { is an open arc in } \mathbb{D}_{U} \text { whose closure } \\
& \text { is a closed } \left.\operatorname{arc} \text { in } \mathbb{D}_{U} \cup\{\alpha, \beta\} \text { from } \alpha \text { to } \beta\right\} .
\end{aligned}
$$

Denote the standard Euclidean topology on $S_{U}^{1}$ by $T_{\mathcal{E}}$ and the topology induced by the Mazurkiewicz metric by $T_{\mathcal{M}}$. We will show that $T_{\mathcal{E}} \subset T_{\mathcal{M}}$ and that $S_{U}^{1}$, with the topology $T_{\mathcal{M}}$, can contain an uncountable discrete subset. Nevertheless we will show that the subspace $\left(\mathcal{A}_{U}, T_{\mathcal{M}}\right)$ is always a separable and complete metric space (see [9, 16, for related results). Since the space $\left(\mathcal{A}_{U}, T_{\mathcal{M}}\right)$ of accessible angles can contain a one-dimensional totally disconnected subspace, the topology $T_{\mathcal{M}}$ on $\mathcal{A}_{U}$ is very different from $T_{\mathcal{E}}$. It is well-known that $\rho_{U}$ is a metric. Hence the easy proof of the following lemma is omitted.

Lemma 5.1. The Mazurkiewicz metric $\rho_{U}$ is a metric on $S_{U}^{1}$.

We will list some observations regarding the Mazurkiewicz metric and angles that correspond to accessible points and two examples.

Remark 5.2. With respect to the usual topology on $S_{U}^{1}$, the subset of accessible angles $\mathcal{A}_{U}$ is a dense subset of $S_{U}^{1}$ of full measure (e.g. [14, Theorem 17.4]).

Lemma 5.3. Every $\alpha \in S_{U}^{1} \backslash \mathcal{A}_{U}$ is isolated in the Mazurkiewicz metric.

Proof. For $\alpha \notin \mathcal{A}_{U}$ and $\alpha \neq \beta$ we always find $\rho_{U}(\alpha, \beta) \geq \operatorname{diam} \Pi(\alpha)>0$. 
Example 5.4. The unit circle equipped with the Mazurkiewicz metric is not necessarily separable. In the light of Lemma 5.3, it suffices to provide an example of a continuum for which uncountably many angles are not accessible angles. A special embedding of the pseudo-arc $P$ in the plane has the property that any two distinct accessible points are in distinct composants (i.e. no proper subcontinuum $X$ of $P$ contains more than one accessible point); cf. [13. Now let $P$ be that continuum and $U=\mathbb{C} \backslash P \cup\{\infty\}$. Let $C$ be any crosscut of $P$. Then $P \subset \overline{\operatorname{Sh}(C)}$, for if this is not the case, then this crosscut would enable us to find a proper subcontinuum of $P$ containing two accessible points. Hence all impressions are equal to $P$. It now follows from a theorem of Collingwood [7] that the set of angles whose principal set is $P$ is a dense, second category subset of $S_{U}^{1}$. Hence $S_{U}^{1} \backslash \mathcal{A}_{U}$ is uncountable and $\left(S_{U}^{1}, \rho_{U}\right)$ contains uncountably many isolated points.

Example 5.5 (Hairy Circle). As a variation on the construction of the Hairy Arc by Aarts and Oversteegen [1, we describe the construction of a continuum $X$ in the plane called the Hairy Circle. There exists an upper semicontinuous map $l: S^{1} \rightarrow[0,1]$ called the length function, such that

(1) for all $z \in \mathbb{C}$ we have $z \in X$ if and only if $z=(1+\lambda) x$, where $x \in S^{1}$ and $0 \leq \lambda \leq l(x)$

(2) the sets $\left\{x \in S^{1}: l(x)>0\right\}$ and $\left\{x \in S^{1}: l(x)=0\right\}$ are both dense in $S^{1}$; and

(3) for each $x \in S^{1}$ with $l(x)>0$ there exist sequences $\left(y_{n}\right)_{n}$ and $\left(y_{n}^{\prime}\right)_{n}$ in $S^{1}$ such that $y_{n} \uparrow x, y_{n}^{\prime} \downarrow x$ and $\lim l\left(y_{n}\right)=\lim l\left(y_{n}^{\prime}\right)=l(x)$.

Let $U$ be the unbounded complementary domain of $X$ and let $\varphi: \mathbb{D} \rightarrow U \cup\{\infty\}$ be a conformal map. In this case it is easily verified that the set of accessible angles $\mathcal{A}=S^{1}$, but we will show that $\left(\mathcal{A}, \rho_{U}\right)$ is homeomorphic to the subset of the plane of all accessible points in $\partial U$. Indeed, the map $\alpha \mapsto \varphi(\alpha)$ is a bijection, and it is clear that whenever $\alpha_{n} \rightarrow \alpha$ in the Mazurkiewicz sense, so that there $\operatorname{are} \operatorname{arcs} J_{n}$ joining $\alpha_{n}$ and $\alpha$ with diam $\varphi\left(J_{n}\right) \rightarrow 0$, then also $x_{\alpha_{n}} \rightarrow x_{\alpha}$ in the plane. To establish continuity of the inverse map, suppose that $x_{\alpha_{n}} \rightarrow x_{\alpha}$, but for some $\varepsilon>0$ and all $\operatorname{arcs} J_{n}$ connecting $\alpha_{n}$ and $\alpha$ we have that diam $\varphi\left(J_{n}\right)>\varepsilon$. Let $x, x_{n} \in S^{1}$ be the unique points for which $x_{\alpha_{n}}=\left(1+l\left(x_{n}\right)\right) x_{n}$ and $x_{\alpha}=(1+l(x)) x$. In particular, $x_{n} \rightarrow x$ in the usual topology on $S^{1}$. By the property for arcs connecting $\alpha_{n}$ and $\alpha$, there also exist $y_{n} \in S^{1}$ so that $l\left(y_{n}\right) \geq l(x)+\varepsilon$ and with $y_{n} \rightarrow x$. Since by upper semicontinuity the set $\left\{y \in S^{1}: l(y) \geq l(x)+\varepsilon\right\}$ is closed in $S^{1}$, we find that $x$ is in the set and hence $l(x) \geq l(x)+\varepsilon$, a contradiction.

The subspace of the plane consisting of endpoints of hairs $\{(1+l(x)) x: x \in$ $S^{1}$ and $\left.l(x)>0\right\}$ is homeomorphic to complete Erdös space (the subspace of $\ell_{2}$, all of whose coordinates are irrational) according to [11. Since that space is totally disconnected, yet one dimensional, the usual topology on the circle and the topology generated by the Mazurkiewicz metric do not necessarily coincide, even when all external rays land.

Let $X$ be the Hairy Circle constructed above and let $h, k: X \rightarrow \mathbb{C}$ be any two embeddings of $X$ in the plane such that in both cases the boundary of the bounded complementary domain of $h(X)$ and of $k(X)$ is a circle. Then it follows from Theorem 4.3 that the homeomorphism $h \circ k^{-1}: k(X) \rightarrow h(X)$ can be extended over the sphere and any two such continua are equivalently embedded in $\mathbb{C}$.

Lemma 5.6. The Mazurkiewicz metric is complete. 
Proof. Suppose we are given a sequence of angles $\left(\alpha_{i}\right)_{i \in \mathbb{N}}$ that is Cauchy with respect to the Mazurkiewicz metric. We may assume that the sequence $\left(\alpha_{i}\right)$ is not constant and, hence, $\operatorname{diam}\left(\Pi\left(\alpha_{i}\right)\right) \rightarrow 0$. Then we can find a subsequence $\left(\alpha_{i_{j}}\right)_{j \in \mathbb{N}}$ and $\operatorname{arcs} J_{j}$ from $\alpha_{i_{j}}$ to $\alpha_{i_{j+1}}$ such that diam $\varphi_{U}\left(J_{j}\right)<2^{-j}$ and $\lim \alpha_{i_{j}}=\alpha$ in $T_{\mathcal{E}}$. Assume $i_{j}=j$ for all $j$. Put $P_{n}=\bigcup_{j \geq n} J_{j}$ and $\varphi_{U}\left(P_{n}\right)=P_{n}^{\prime}$. Then $\operatorname{diam}\left(P_{n}^{\prime}\right) \rightarrow 0$, and we may assume that $\lim P_{n}^{\prime}=x$. If $x \notin \partial U$, we would be able to find an open neighbourhood $N$ of $x$ in the sphere such that $\bar{N} \cap \partial U=\emptyset$, which is impossible since for some $m \in \mathbb{N}$ we have that $P_{m} \subset N$. We can approximate each $P_{n}$ by an open $\operatorname{arc} A_{n}$ whose closure is an arc joining the points $\alpha_{n}$ and $\alpha$ such that $\operatorname{diam}\left(\varphi_{U}\left(A_{n}\right)\right) \leq \operatorname{diam}\left(P_{n}^{\prime}\right)+1 / n$. Hence $\rho_{U}\left(\alpha_{n}, \alpha\right) \rightarrow 0$ and moreover $\alpha \in \mathcal{A}_{U}$. Then $\lim \alpha_{i}=\alpha$ in $T_{\mathcal{M}}$, as required.

Note that by Lemma 5.3 the subspace $\mathcal{A}_{U}$ of $\left(S_{U}^{1}, T_{\mathcal{M}}\right)$ is a closed subset. Hence we have the following corollary.

Corollary 5.7. The subspace $\mathcal{A}_{U}$ with the Mazurkiewicz metric is a complete metric space.

Lemma 5.8. The topology generated by the Mazurkiewicz metric on $S_{U}^{1}$ is stronger than the original topology.

Note that we do not intend to show that the topology is strictly stronger. Indeed, take $X=S^{1}$.

Proof. If $\alpha \notin \mathcal{A}_{U}$, then the set $\{\alpha\}$ is open with respect to the Mazurkiewicz metric by Lemma 5.3. so let $\alpha \in \mathcal{A}_{U}$ be arbitrary and consider some open interval $N \subset S_{U}^{1}$ containing $\alpha$. Then we can find angles $\beta, \beta^{\prime} \in \mathcal{A}_{U}$ with $\beta<\alpha<\beta^{\prime}$ and $\varphi_{U}(\beta) \neq \varphi_{U}(\alpha) \neq \varphi_{U}\left(\beta^{\prime}\right) \neq \varphi_{U}(\beta)$ such that the interval $\left(\beta, \beta^{\prime}\right) \subset N$. Let $r<\infty$ be such that $X \subset B(O, r / 2)$, the closed ball about $O$ of radius $r / 2$. Define $\varepsilon=d\left(R_{\alpha} \cap B(O, r),\left(R_{\beta} \cup R_{\beta^{\prime}}\right) \cap B(O, r)\right)$ and note that $\varepsilon>0$. Let $\gamma \notin N$. It suffices to show that $\rho_{U}(\alpha, \gamma) \geq \varepsilon$. For this, note that there has to exist an arc $J$ connecting $\alpha$ and $\gamma$ within $B(O, r)$ and that the image of such an arc intersects either $R_{\beta}$ or $R_{\beta^{\prime}}$ by an order argument. Hence the image under $\varphi_{U}$ always has diameter at least $\varepsilon$ and $\rho_{U}(\alpha, \gamma) \geq \varepsilon$.

Corollary 5.9. If $\operatorname{dim}\left(\mathcal{A}_{U}, T_{\mathcal{E}}\right)=0$, then $\left(\mathcal{A}_{U}, T_{\mathcal{M}}\right)$ is totally disconnected.

Proposition 5.10. The subspace $\mathcal{A}_{U}$ of $S_{U}^{1}$ equipped with the Mazurkiewicz metric is separable.

Proof. We consider the hyperbolic lamination $\mathcal{H}$ of $U$ introduced in the preliminaries. Let $N$ be the collection of all centers $c \in \mathcal{C}$ such that for the maximal ball $B(c, r(c))=B, \bar{B} \cap \partial U$ contains nondegenerate $\operatorname{arcs} I_{n}^{c}$. Then $N$ is countable and $J=\bigcup_{c \in N} \bigcup_{n} \varphi_{U}^{-1}\left(I_{n}^{c}\right)$ is a countable union of subarcs of $S_{U}^{1}$. Since on each $\operatorname{arc} \varphi_{U}^{-1}\left(I_{n}^{c}\right)$ the Mazurkiewicz topology and the normal subspace topology of $S_{U}^{1}$ coincide, $J$ contains a countable dense subset. Hence we only need to consider $\mathcal{A}_{U} \backslash J$.

It follows from Corollary 2.4 that if $\mathcal{H}_{n}:=\{\ell \in \mathcal{H} \mid \operatorname{diam}(\ell) \geq 1 / n\}$, then the collection $\mathcal{H}_{n}^{*}$ of the closures of all hyperbolic leaves in $\mathcal{H}_{n}$ is a compact subset of the hyperspace of closed subsets $2^{\mathbb{C}^{*}}$ of the sphere. Hence there exists a countable collection $\mathcal{C}_{n}$ of hyperbolic leaves in $\mathcal{H}_{n}$ such that the closure of every hyperbolic leaf in $\mathcal{H}_{n}$ is the limit of a sequence of hyperbolic leaves in $\mathcal{C}_{n}$. Let $\mathcal{L}_{n}$ be the 
collection of all leaves of the form $\varphi_{U}^{-1}(\ell)$ for $\ell \in \mathcal{C}_{n}$ and let $E$ be the set of all endpoints of leaves in $\bigcup_{n} \mathcal{L}_{n}$.

Then $E$ is a countable subset of $\mathcal{A}_{U}$. We claim that $E$ is dense in $\mathcal{A}_{U} \backslash J$. To see this choose $\theta \in \mathcal{A}_{U} \backslash J$. If $\varphi_{U}(\theta)$ is the endpoint of a hyperbolic leaf $\ell \in \mathcal{H}$, then there exist $n$ and a sequence $\ell_{i} \in \mathcal{C}_{n}$ such that $\lim \ell_{i}=\bar{\ell}$. Hence one endpoint, say $x_{i}$ of $\ell_{i}$, must converge to $\varphi_{U}(\theta)$. Note that if the hyperbolic leaf $\ell_{i}$ is contained in the maximal ball $B_{i}$, then $\lim B_{i}=B$ is a maximal ball containing $\ell$ (see [5, Section 4]). It now follows easily that $\varphi_{U}^{-1}\left(x_{i}\right)$ must converge to $\theta$ in the Mazurkiewicz metric. On the other hand, suppose that $\varphi_{U}(\theta)$ is not the endpoint of any hyperbolic leaf in $\mathcal{H}$. Consider the external ray $R_{\theta}$ which runs from $\infty$ to $\varphi_{U}(\theta)=x_{\theta}$. By Theorem 2.2, there is either a single hyperbolic gap $\Gamma$ of $\mathcal{H}$ which contains a terminal segment of $R_{\theta}$, or there exists a sequence of hyperbolic leaves $\ell_{i} \in \mathcal{H}$ such that $\lim \ell_{i}=x_{\theta}$. In the latter case, since each $\ell_{i}$ is approximated by hyperbolic leaves in some $\mathcal{C}_{n}$, there exists a sequence of points $\theta_{i} \in E$ such that $\theta=\lim \theta_{i}$. In the first case, since $\theta \in \mathcal{A}_{U} \backslash J, \varphi_{U}(\theta)$ is not the endpoint of a hyperbolic leaf and $x_{\theta}$ is contained in the closure of a single hyperbolic gap $\Gamma$, there exists a countable sequence of hyperbolic leaves $\ell_{i} \subset \partial \Gamma$ which converges to $\varphi_{U}(\theta)$. By construction of the collections $\mathcal{C}_{n}$, the hyperbolic leaves $\ell_{i}$ are approximated by hyperbolic leaves from $\bigcup_{n} \mathcal{C}_{n}$. It now follows easily that also in this case there exists a sequence of points $\theta_{i} \in E$ such that $\theta=\lim \theta_{i}$ in the Mazurkiewicz metric. This completes the proof.

\section{ACKNOWLEDGEMENT}

We are indebted to the referee for several useful comments.

\section{REFERENCES}

1. J. M. Aarts and L. G. Oversteegen, The homeomorphism group of the hairy arc, Compositio Math. 96 (1995), no. 3, 283-292. MR1327147 (96b:54056)

2. J. Aarts, G. Brouwer and L. Oversteegen, Centerlines of regions in the sphere, Topology and its Appl. 156 (2009), no. 10, 1776-1785. MR2519213

3. R. H. Bing and M. Starbird, Linear isotopies in $E^{2}$, Trans. Amer. Math. Soc. 237 (1978), 205-222. MR0461510 (57:1495)

4. A. M. Blokh and L. G. Oversteegen, A fixed point theorem for branched covering maps of the plane, Fund. Math. 206 (2009), 77-111. MR2576262

5. A. M. Blokh, R.J. Fokkink, J. C. Mayer, L. G. Oversteegen, and E. D. Tymchatyn, Fixed point theorems for plane continua with applications, arXiv:1004.0214.

6. G. A. Brouwer, Green's functions from a metric point of view, Ph.D. dissertation, University of Alabama at Birmingham, 2005.

7. E. F. Collingwood, A theorem on prime ends, J. London Math. Soc. 31 (1956), 344-349. MR 0080158 (18:201e)

8. R. D. Edwards and R. C. Kirby, Deformations of spaces of imbeddings, Ann. of Math. (2) 93 (1971), 63-88. MR0283802 (44:1032)

9. D. B. A. Epstein, Prime ends, Proc. London Math. Soc. (3) 42 (1981), no. 3, 385-414. MR 614728 (83c:30025)

10. Curves on 2-manifolds and isotopies, Acta Math. 115 (1966), 83-107. MR0214087 $(35: 4938)$

11. K. Kawamura, L. G. Oversteegen, and E. D. Tymchatyn, On homogeneous totally disconnected 1-dimensional spaces, Fund. Math. 150 (1996), 97-112. MR1391294 (97d:54060)

12. R. S. Kulkarni and U. Pinkall, A canonical metric for Möbius structures and its applications, Math. Z. 216 (1994), no. 1, 89-129. MR.1273468(95b:53017)

13. W. Lewis, Embeddings of the pseudo-arc in $E^{2}$, Pacific J. Math. 93 (1981), no. 1, 115-120. MR621602 (82h:54056) 
14. J. Milnor, Dynamics in one complex variable, third ed., Annals of Mathematics Studies, vol. 160, Princeton University Press, Princeton, NJ, 2006. MR2193309 (2006g:37070)

15. L. G. Oversteegen and E. D. Tymchatyn, Extending isotopies of planar continua, Annals of Mathematics 172 (2010), 2105-2133.

16. P. Seibert, Über die Randstrukturen von Überlagerungsflächen, Math. Nachr. 19 (1958), 339352. MR0105497 (21:4238)

17. C. Pommerenke, Boundary behaviour of conformal maps, Grundlehren der Mathematischen Wissenschaften [Fundamental Principles of Mathematical Sciences], vol. 299, Springer-Verlag, Berlin, 1992. MR,1217706 (95b:30008)

18. L. Siebenmann, The Osgood-Schoenflies Theorem revisited, Russian Math. Surveys 60 (2005), 645-672. MR2190924 (2007e:57011)

19. W. P. Thurston, On the geometry and dynamics of iterated rational maps, Complex dynamics: families and friends, A K Peters, Wellesley, MA, 2009, pp. 3-137. MR 2508255

Department of Mathematics, University of Alabama at Birmingham, Birmingham, Alabama 35294-1170

E-mail address: overstee@math.uab.edu

Faculteit der Exacte Wetenschappen, Afdeling Wiskunde, Vrije Universiteit, De Boelelaan 1081A, 1081 HV Amsterdam, The Netherlands

E-mail address: kivalken@few.vu.nl 